\title{
México íuna frontera Vertical? Políticas de CONTROl del tránsito MIGRATORIO IRREGULAR Y SUS RESULTADOS, 2007-2016
}

\author{
Mexico, a Vertical Border? Policies to Control Irregular Migration and Its Results, 2007-2016 \\ Eduardo Torre-Cantalapiedra \\ José Carlos Yee-Quintero
}

Resumen: El objetivo de este trabajo es analizar en qué medida la política migratoria mexicana ha convertido el territorio nacional en una extensa frontera para los migrantes irregulares en tránsito a Estados Unidos, denominada "frontera vertical". Para ello, se examinan las políticas de control mexicanas, incluyendo la legislación sobre el tema, y se analizan algunos resultados mediante el uso de diversas fuentes de datos administrativos, con especial atención en el período 2007-2016. Se concluye que la estrategia de contención supuso la generación de una "frontera vertical" estable, y que la forma de implementar dicha estrategia generó un comportamiento diferenciado en las prácticas de control migratorio.

Palabras clave: migración internacional, politicas migratorias mexicanas, migración en tránsito, fronteras, centroamericanos.

Abstract: The objective of this paper is to analyze the extent to which Mexican migration policy has made Mexico an extensive border for irregular migrants in transit to the United States, the so-called "vertical border." To this end, it examines Mexican control policies, including relevant legislation, with emphasis on the last decade (2007-2016). In addition, the policy implemented over the same period and some results are analyzed using various administrative data sources. We conclude that during the past decade, the general strategy of containment created a stable "vertical border," and the way that strategy was implemented generated a differentiated behavior in migratory control practices.

Keywords: international migration, Mexican migration policies, transit migration, borders, Central Americans.

Eduardo Torre Cantalapiedra. Doctor en Estudios de Población por el Colegio de México, México. Investigador posdoctoral en el Instituto de Investigaciones Jurídicas de la Universidad Nacional Autónoma de México, México. Temas de especialización: política migratoria, familia y políticas públicas, patrones migratorios y migración internacional. Correo electrónico: e.torreca@gmail.com.

José Carlos Yee Quintero. Maestro en Estudios de Población por El Colegio de la Frontera Norte, México. Tutor académico de la
Especialidad en Migración Internacional de El Colegio de la Frontera Norte, México, y coordinador de la Casa del Migrante en Tijuana A.C., México. Temas de especialización: migración internacional, migración de retorno y reinserción social, y violencia multidimensional. Correo electrónico: jcyeel@gmail.com.

Enviado a dictamen: 31 de octubre de 2017

Aprobación: 20 de marzo de 2018

Revisiones: 1 


\section{Introducción}

$\mathrm{D}$ esde la década de los noventa, la migración centroamericana en tránsito por México ha sido un fenómeno que por su volumen — con flujos anuales de cientos de miles de transmigrantes-, alto predominio de la irregularidad y destacada tendencia a presentar abusos y violencia ha suscitado el interés de políticos, de académicos y de la sociedad civil. Desde entonces se ha producido un apasionado debate público respecto a cómo el gobierno mexicano debe interactuar con estos flujos migratorios, así como las medidas de contención o protección que deberían, o no, ser implementadas. ${ }^{1}$

Simultáneamente, otra interrogante que ha despertado un interés similar se refiere a cómo ha gestionado el gobierno mexicano los flujos migratorios centroamericanos en tránsito irregular por el territorio nacional. Desde el ámbito académico, en diversas investigaciones se han analizado en mayor o menor detalle, en ocasiones de forma tangencial, las políticas migratorias de control del tránsito migratorio conducidas por el gobierno de México. Así, las normativas y políticas al respecto y su implementación han sido muy estudiadas, y se evidenció el inadecuado actuar gubernamental en relación con el respeto irrestricto de los derechos de los migrantes. ${ }^{2}$

Otro aspecto destacado por los académicos, en cuanto a la manera en que se lleva a cabo la tarea de contención de migrantes en tránsito, es que México ha convertido su territorio en una extensa frontera para los migrantes en tránsito, y como resultado los migrantes centroamericanos que cruzan el país con destino a Estados Unidos están continuamente expuestos a ser detenidos y expulsados de diversas formas contempladas en la legislación mexicana; ${ }^{3}$ esta implementación de la política migratoria en el país ha sido denominada por varios autores como "frontera vertical". ${ }^{4}$

En otras palabras, las acciones y los operativos que se llevan a cabo para controlar los flujos se han extendido a lo largo de todo el territorio nacional debido a la creciente importancia de México como país de tránsito
MÉXICO ¿UNA FRONTERA VERTICAL? POLÍTICAS DE CONTROL DEL TRÁNSITO MIGRATORIO IRREGULAR Y SUS RESULTADOS, 2007-2016

y receptor de migrantes (Anguiano y Trejo, 2007); en este sentido, destaca cómo las fronteras geopolíticas son espacios de ejercicio del poder y filtros selectivos de la migración (Silva Hernández, 2015).

Cabe mencionar que la labor de contención realizada por México, al igual que la de otros países considerados de tránsito de migrantes - por ejemplo, Marruecos-, debe ponerse en relación con las políticas inmigratorias del Estado que finalmente recibe a los migrantes, en este caso, Estados Unidos. Lo anterior se debe a que, como señalan algunos autores, se puede apreciar una tendencia de los "países desarrollados" a la "externalización" de sus fronteras - expresión que se retoma de Baggio (2010) - , por ejemplo, tratando de incidir en las políticas migratorias de los países de tránsito para evitar que migrantes y refugiados lleguen a sus territorios.

El objetivo de este trabajo es analizar la política y las prácticas de control de la migración centroamericana ${ }^{5}$ en tránsito irregular por el territorio del país en la década comprendida entre 2007 y 2016, y en qué medida produjeron, o no, una "frontera vertical" para controlar la migración en tránsito. Para lograrlo, en un primer apartado se examinan la estrategia y las políticas de control -incluyendo las normativas - respecto a la inmigración indocumentada en tránsito por México durante los sexenios de Felipe Calderón Hinojosa (2006-2012) y de Enrique Peña Nieto (2012-2018). ${ }^{6}$ Si bien se hace énfasis en el periodo señalado, el análisis se remonta a la década de los ochenta para contextualizar históricamente el fenómeno. Con este propósito se realizó una extensa revisión de la literatura académica y de artículos de prensa, y se llevaron a cabo entrevistas con académicos, miembros de organizaciones no gubernamentales y funcionarios públicos.

En un segundo apartado, haciendo uso de datos administrativos de libre acceso y de otros obtenidos mediante consultas a través de la Plataforma Nacional de Transparencia de México, ${ }^{7}$ se analizan las prácticas de control migratorio a lo largo del territorio del país desde una perspectiva cuantitativa. Son tres los rubros que se analizan por regiones - Norte, Centro, Sur y Frontera Sur-: 1) las revisiones migratorias, 2) las 
EDUARDO TORRE-CANTALAPIEDRA

JOSÉ CARLOS YEE-QUINTERO
MÉXICO ¿UNA FRONTERA VERTICAL? POLÍTICAS DE CONTROL DEL TRÁNSITO MIGRATORIO IRREGULAR Y SUS RESULTADOS, 2007-2016 presentaciones ante la autoridad migratoria ${ }^{8}$ y 3) las estaciones migratorias y estancias provisionales.

\section{Políticas mexicanas para el control del tránsito de inmigrantes indocumentados a lo largo del territorio}

Entre las leyes, reglamentos y medidas referidas al control migratorio durante la década analizada destacan los siguientes factores: 1) que la estrategia general de control de la migración en tránsito fue establecida a comienzos del siglo XXI con el Plan Sur (2001) y con el establecimiento de centros de detención a lo largo de todo el territorio nacional; 2) la tensión entre las perspectivas de seguridad nacional y los derechos humanos de los migrantes en las políticas de control del tránsito migratorio; 3) la importancia de los medios y recursos dedicados a la implementación de aquéllas, y 4) la carencia de información relevante para el análisis de las políticas de contención y la falta de precisión de la existente.

Hacia una estrategia de contención migratoria en México: el Plan Sur, la expansión de las estaciones migratorias yel paradigma de la seguridad nacional

Hasta mediados del decenio de los años setenta los flujos migratorios internacionales en Centroamérica se producían mayoritariamente dentro de la propia región (Castillo, 2001). A partir de entonces, primero por razones ligadas a los conflictos políticos y a las confrontaciones armadas, y posteriormente por razones de tipo económico — crisis económicas - y social — aumento de la violencia-, fueron creciendo los flujos de migrantes centroamericanos indocumentados en tránsito por México con destino a Estados Unidos. La cifra de migrantes en tránsito captados por los registros administrativos ${ }^{9}$ superó una decena de miles de eventos de detención en 1980 y fueron más de cien mil en 1990 (Castillo, 2001).

El referente legislativo para la política respecto al flujo de inmigrantes indocumentados fue la Ley General de Población (LGP) de 1974, que experimentó varias reformas, entre ellas tres cambios relevantes durante la década de los noventa. Según esta ley, correspondía a la Secretaría de Gobernación (SEGOB), entre otros asuntos, "vigilar la entrada y salida de los nacionales y extranjeros, y revisar la documentación de los mismos" (Congreso de la Unión, 1974: art. $7^{\circ}$ II). En su artículo 41 contemplaba la calidad de transmigrante ${ }^{10}$ — dentro de la categoría de "No migrante"-, pero su reglamento contenía un requisito que pocos migrantes en tránsito podrían cumplir: estar en posesión de un permiso de admisión al país que se dirigen. Asimismo, la Ley General de Población contemplaba sanciones muy duras para aquellos que se encontrasen de manera ilegal en el país, a los que se consideraba criminales.

Hasta la década de los ochenta, las acciones de aplicación de las leyes de migración en México, que eran significativamente poco numerosas, fueron gestionadas discrecionalmente y caso por caso por la Dirección General de Servicios Migratorios dependiente de la Secretaria de Gobernación (Alba y Castillo, 2012). En definitiva, México no contó con una política gubernamental respecto a estos flujos hasta los años noventa, ${ }^{11}$ cuando se produjo un incremento acelerado de los flujos de migrantes en tránsito a Estados Unidos (Castillo, 2005).

En 1993 se creó el Instituto Nacional de Migración (INM), órgano desconcentrado del gobierno federal, pero subordinado a la SEGOB. Éste sustituía a la Dirección General de Servicios Migratorios, y fue facultado para llevar a cabo las atribuciones contenidas en la Ley General de Población respecto a la materia de migración (Alba y Castillo, 2012). Además, durante los noventa se construyeron o adecuaron más de una veintena de instalaciones para el alojamiento de inmigrantes indocumentados.

En 1996 se produjo una reforma a la Ley General de Población que introdujo, entre otros, los artículos 151 y 152, que facultan la "Revisión migratoria en rutas o puntos provisionales distintos a los establecidos" (Congreso de la Unión, 1974, reformado en 1996: art. 151.V) y autorizan el aseguramiento de extranjeros en caso de que ameriten su expulsión por alguna infracción a lo dispuesto en la ley, su reglamento u 
otras disposiciones aplicables (Congreso de la Unión, 1974, reformado en 1996: art. 152). Esto marcó el inicio de una nueva era en las mecánicas de contención y la implementación del control migratorio en México.

En los primeros compases del sexenio de Vicente Fox Quesada (2000-2006) se desarrolló y puso en marcha el Plan Sur, ${ }^{12}$ programa destinado al control de la migración. En éste se consideraba realizar la contención migratoria en círculos graduales que llegaban hasta el Istmo de Tehuantepec.

El primer control estaría a cargo de las delegaciones del Instituto Nacional de Migración en los estados de Chiapas y Tabasco; mientras que del segundo las delegaciones de Oaxaca y Veracruz. En el primero se plantearon cinco puntos de inspección y el segundo cinturón de seguridad más al norte abarcaría seis (Silva Quiroz, 2014: 49).

Se trató de una estrategia regional que dio significado a la distribución espacial de las delegaciones del INM (Casillas, 2016).

Oficialmente el Plan Sur fue sustituido en 2003 por el proyecto Fortalecimiento de las Delegaciones Regionales de la Frontera sur (García y Tarrío, 2008; Silva Quiroz, 2014). Pero esto no quiere decir que la estrategia de contención en el Istmo de Tehuantepec, ya establecida en años anteriores, dejara de estar vigente, aun cuando no fuese una política muy definida y se enfocara al uso de la fuerza.

Entre los años 2000 y 2005 el número de estaciones migratorias a nivel nacional fue incrementado de manera significativa, de 25 a 52 unidades; si bien en ambos años el mayor número de estaciones se concentraba en la región sureste del país, en 2005 se observó una mayor presencia a lo largo de todo el territorio nacional (Casillas, 2007, 2016). De acuerdo con la estrategia establecida, los migrantes indocumentados pueden ser detectados y llevados a una estación migratoria para su posterior deportación en cualquier parte, a lo largo de todo el territorio nacional, no sólo en la frontera sur. Asimismo, esta estrategia supone detener el flujo de migrantes en tránsito hacia Estados Unidos en el Istmo de Tehuantepec o a lo largo de la ruta migratoria, más que hacerlo sobre la línea fronteriza México-Guatemala (Torre y Schiavon, 2016). Esto no sólo modificó la implementación de la normatividad sobre control migratorio, sino que fue alterando los límites del concepto mismo de control inmigratorio en México. En este punto de la evolución del control migratorio puede observarse un enfoque hacia el transmigrante no sólo al ingresar a México, sino en el trayecto, en puntos cada vez más cercanos a su salida discrecional del país.

Otro cambio importante en este tema se produjo tras el 11 de septiembre de 2001. El principal impacto de los eventos ocurridos ese fatídico día fue que tanto en Estados Unidos, como en México, el diseño de las políticas públicas pasó a ubicarse bajo un paradigma de seguridad nacional. Si bien esa tendencia había comenzado a gestarse en años previos, tras la caída de las torres gemelas se cristalizó esa inclinación. Uno de los hechos más relevantes en este sentido fue la inclusión del INM como instancia de seguridad nacional en el año 2005, al darse al instituto un lugar en el Consejo Nacional de Seguridad Pública y acceso a diversos beneficios.

Ese mismo año, el INM publicó su "Propuesta de política migratoria integral en la Frontera Sur de México", que contemplaba cuatro líneas estratégicas: 1) facilitación de los flujos migratorios documentados que tienen como destino temporal y definitivo los estados de la frontera sur de México; 2) protección de los derechos de los migrantes que se internan por la frontera sur de México;3) contribución a la seguridad en la frontera sur de México, y 4) actualización de la gestión de los flujos y de la legislación migratoria, tomando en consideración las particularidades del fenómeno de la frontera sur (INM, 2005: 6). Pese a no ser un documento preceptivo, sí permite revisar el debate sobre cuáles han sido las actuaciones del gobierno mexicano en cuanto a la gestión de la inmigración y da pie a un debate más amplio sobre cómo debería, o no debería, ser dicha actuación. 
EDUARDO TORRE-CANTALAPIEDRA

Mucha retórica sobre los derechos de los migrantes, pocas novedades en la estrategia de contención y algunos cambios

A partir de la administración calderonista el paradigma de los derechos humanos, que ya había tenido cierta presencia desde mediados de los noventa y que fue extensamente reivindicado por las organizaciones de la sociedad civil, ${ }^{13}$ fue predominante en la agenda política mexicana sobre migración junto al omnipresente paradigma de la seguridad.

Durante el sexenio de gobierno de Felipe Calderón Hinojosa -2006-2012 - dos temas principales de la agenda interior y exterior fueron: la seguridad nacional y el combate al crimen organizado (Velázquez y Domínguez, 2013). Siendo esto así, México incorporó la migración como un asunto de seguridad, dando la idea de que esta materia debía ser tratada de manera similar al narcotráfico y a otros delitos conexos (Urbano, 2012: 78), justificando y reforzando la idea de necesidad de un férreo control migratorio.

Al mismo tiempo, se produjeron avances en cuanto al reconocimiento de los derechos humanos de los migrantes, que ocuparon un lugar prominente en el Plan Nacional de Desarrollo 2007-2012. En el objetivo 10 de dicho plan, "construir una nueva cultura de la migración", se señala que hay que "garantizar [...] la protección de los derechos de los migrantes" (Gobierno de los Estados Unidos Mexicanos, 2007: 307). La primera estrategia en pro del logro de dicho objetivo era: "garantizar el respeto y protección de los derechos humanos de los migrantes de otros países en suelo mexicano" (Gobierno de los Estados Unidos Mexicanos, 2007: 307).

El debate público y académico ha girado en torno a la cuestión de si es posible tener una política migratoria inspirada al mismo tiempo en el paradigma de seguridad nacional que busca el control, y en el paradigma de los derechos humanos que pretende la protección de los migrantes. Los cambios en las leyes y políticas migratorias de esta década deben ser analizados desde estas dos visiones, que muchos autores consideran en conflicto.

En 2007 y años posteriores, de acuerdo con una agenda de securitización "binacional", las acciones para
MÉXICO ¿UNA FRONTERA VERTICAL? POLÍTICAS DE CONTROL DEL TRÁNSITO MIGRATORIO IRREGULAR Y SUS RESULTADOS, 2007-2016

el control de flujos migratorios se vieron "apoyadas" por la Iniciativa Mérida (Silva Quiroz, 2014).

Enésta, el gobiernode Estados Unidos secomprometió a proporcionar asistencia a México para: “(1) romper el poder y la impunidad de las organizaciones criminales; (2) reforzar la frontera, y los controles aéreos y marítimos; (3) mejorar la capacidad de los sistemas de justicia en la región, y (4) reducir la actividad de pandillas y disminuir la demanda local de drogas (Ribando y Finklea, 2013, citados por Silva Quiroz, 2014: 51).14

En el año 2008 se produjo un gran avance legislativo respecto a los derechos de los migrantes y una reforma a la Ley General de Población en la que se despenalizaba la inmigración indocumentada. De acuerdo con González-Murphy y Koslowski (2011), este cambio en la legislación migratoria mexicana, como otros anteriores y posteriores -incluso algunos que no se habían producido cuando escribieron su artículo, por ejemplo, la Ley de Migración de 2011-, tiene su explicación, al menos en parte, en la búsqueda del gobierno mexicano de lograr la congruencia entre las exigencias del gobierno de Estados Unidos en cuanto a políticas migratorias, y la legislación mexicana en la materia.

Sin embargo, la despenalización de la migración irregular no parece traducirse en una mejor situación para los migrantes; la masacre de San Fernando Tamaulipas en el año 2010 evidenció como nunca antes las violaciones de derechos humanos a las que estaban expuestos los migrantes en tránsito por México. A este trágico suceso le había antecedido un reporte de la Comisión Nacional de Derechos Humanos (CNDH) de 2009 en el que se indicaba: "que el número de migrantes que fueron víctimas de privación de su libertad fue de 9,758 personas, es decir, más de 1,600 secuestrados por mes" (CNDH, 2009: 12).

Estos hechos, y su trascendencia mediática, posiblemente aceleraron la aprobación de una ley que llevaba conformándose desde comienzos de siglo. Así, en el año 2011 se produjo un cambio normativo 
fundamental con la publicación de la Ley de Migración, que se vio refrendado con la aprobación posterior de su reglamento (2012). Se trata de una ley que se enfoca de manera específica en materia migratoria, principalmente en la inmigración y la migración en tránsito, y en ella ocupan un lugar destacado los derechos de los migrantes. Así, en el artículo 2 de la misma se señala, como un principio en el que debe sustentarse la política migratoria del Estado, que existirá un: "Respeto irrestricto de los derechos humanos de los migrantes, nacionales y extranjeros, sea cual fuere su origen, nacionalidad, género, etnia, edad y situación migratoria" (Congreso de la Unión, 2017: art. 2). Sin embargo, diversos autores señalan que varias de sus disposiciones serían en gran medida ineficaces en la práctica (Urbano, 2012; Silva Quiroz, 2015; Espino y Doncel de la Colina, 2016).

Los artículos 18 y 19 de la Ley de Migración atribuyen a la SEGOB la labor de formular y dirigir la política migratoria, y al INM la: “ejecución, control y supervisión de los actos realizados por las autoridades migratorias en territorio nacional, así como la instrumentación de políticas en la materia, con base en los lineamientos que expida la misma Secretaría" (Congreso de la Unión, 2017: art. 19). Por otra parte, en ella se especifica que el presidente de la República nombra al titular de la Subsecretaría de Población, Migración y Asuntos Religiosos, entidad encargada de la política migratoria, y como titular del INM a un Comisionado, lo que en la práctica dificulta las relaciones entre ambos (Insyde, 2013).

Al igual que la Ley General de Población, la Ley de Migración (2011) permite: "llevar a cabo revisiones de carácter migratorio dentro del territorio nacional a efecto de comprobar la situación migratoria de los extranjeros" (art. 97). Asimismo:

[...] en aras de cumplir con los objetivos, la regulación migratoria otorga a las Delegaciones Federales gran autonomía para su regulación interna, lo cual ha ocasionado que la gestión en cada Delegación varíe según el estilo del titular, y que el sector central tenga poca injerencia sobre las acciones de los delegados (Insyde, 2013: 30).
En el Plan Nacional de Desarrollo 2013-2018, al igual que en su antecesor, se da gran importancia a los derechos de los migrantes, al reconocer como fundamental la protección a los derechos de los migrantes y de sus familias. Asimismo, pone en marcha la elaboración de un programa que fungiría: "como el instrumento programático para el diseño, implementación, seguimiento y evaluación de la política y la gestión migratoria" (Gobierno de los Estados Unidos Mexicanos, 2013: 153). Éste fue el Programa Especial de Migración 2014-2018, entre cuyos objetivos y acciones se incluyeron numerosas cuestiones relativas a los derechos de los migrantes, además de diversas alusiones a puntos de seguridad fronteriza. En este sentido:

[...] fuentes de la CNS [Comisión Nacional de Seguridad] precisaron que habrá conexiones o nodos carreteros y vigilancia especial en puntos costeros; por ejemplo, en la zona de Huixtla, Suchiate, Arriaga, Trinitaria, Comitán, Benemérito de las Américas y Palenque; un segundo cinturón de seguridad en Frontera (Tabasco), y el tercero en el Istmo, hacia áreas específicas de Veracruz como Las Choapas y Cosoleacaque (Martínez y Castillo, 2014).

Otro punto clave en este encadenamiento de cambios en el control migratorio mexicano se produjo el 7 de julio de 2014, cuando Peña Nieto anunció el Programa Integral Frontera Sur, el cual tiene como finalidad la protección de los migrantes que transitan por el país con destino a Estados Unidos. Este programa ha destacado por su falta de transparencia (Díaz, 2016) y de planeación, por lo que es otro buen ejemplo de la imposibilidad de una aproximación precisa a la manera en que las políticas migratorias están siendo implementadas. Villafuerte Solís y García Aguilar (2015) incluso señalan que, inmerso en la denominada "crisis humanitaria de los niños migrantes mexicanos y centroamericanos" en el año 2014, México se alineó con los intereses de la política inmigratoria de Estados Unidos, de la misma forma que los países del triángulo norte centroamericano, y, siguiendo lógicas de seguridad nacional, el gobierno 
mexicano amplió considerablemente su labor de contención del flujo de migrantes centroamericanos con destino al país del norte.

Pese a todos los cambios en este tema durante el último decenio, los abusos y la violencia en contra de los migrantes han permanecido y, en contra de los discursos que se han tejido a la par de las decisiones legislativas —en pro de la protección de los migrantes y el respeto de sus derechos-, aparentemente han ido en aumento como lo demuestran numerosos informes e investigaciones. ${ }^{15}$

\section{Prácticas de control migratorio a lo largo del territorio mexicano y resultados}

En este apartado se analizan las prácticas respecto al control de la migración en tránsito y sus resultados utilizando diferentes fuentes de datos administrativos de acceso público, así como información recabada a través de solicitudes realizadas en la Plataforma Nacional de Transparencia. Son tres las variables utilizadas: 1) las revisiones migratorias, 2) las presentaciones y 3) las estaciones migratorias y estancias provisionales.

\section{Cuestiones metodológicas}

Las fuentes de datos utilizadas, estadísticas del INM, presentan algunas insuficiencias y limitaciones que se deben tener en cuenta. En primer lugar, las estadísticas en materia migratoria han sido históricamente deficientes, y aunque la mejoría ha sido notable, su falta de precisión continúa siendo una limitante en la actualidad. En su análisis, Casillas (2012) concluye que hay serias dudas sobre considerar que las cifras del INM, que indican una disminución del flujo transmigratorio detenido por la autoridad mexicana a partir del año 2007, sean veraces. En el año 2014 la auditoría de desempeño del INM en materia de política migratoria, realizada por la Auditoría Superior de la Federación, puso de manifiesto errores en la exhibición de las actuaciones realizadas. De acuerdo con el órgano auditor gubernamental, mientras que las revisiones migratorias realizadas en el año 2014, ascendieron, según los registros, a 25383 , en los consolidados de información se recogían tan sólo 17284 . Asimismo, encontraron errores en el reporte de las cifras de presentados y en otras estadísticas sobre las actuaciones planificadas y realizadas por el INM.

En segundo lugar, en lo que respecta a las presentaciones ante la autoridad migratoria, un problema añadido es que éstas corresponden a eventos, y no a personas. Esto quiere decir que un mismo migrante en tránsito puede sufrir durante el mismo periodo más de un evento de este tipo, lo que impactaría en las cifras. Tal característica implica que posibles variaciones en el comportamiento migratorio, como la disminución o el aumento de intentos de cruce por migrante, puede alterar el volumen de las presentaciones sin que sea indicador fiable del flujo en cuanto a personas. De igual forma, el sitio de captura puede reportar que se capturó a un migrante indocumentado establecido, y que éste fue considerado como un transmigrante. Casillas señala que la razón o las razones por las que una persona puede ser detenida varias veces son: "experiencia migratoria; concurso y éxito de una red de acompañamiento, y éxito de la política gubernamental en la detección y detención del migrante indocumentado" (Casillas, 2012: 35).

En cualquier caso, dado que el presente análisis se centra en la implementación de la política de contención a lo largo del territorio mexicano, y no en la precisión absoluta de las cifras, se considera que las estadísticas del INM permiten esbozar grosso modo un panorama general. Para este análisis nos basaremos en una regionalización desarrollada en un trabajo previo (Yee y Torre, 2016) que agrupa las entidades federativas en cuatro regiones: 1) Frontera Sur: Campeche, Chiapas, Quintana Roo y Tabasco; 2) Sur: Oaxaca, Veracruz y Yucatán; 3) Centro: Aguascalientes, Colima, Ciudad de México, Guanajuato, Guerrero, Hidalgo, Jalisco, México, Michoacán, Morelos, Nayarit, Puebla Querétaro, San Luis de Potosí, Tlaxcala y Zacatecas, y 4) Norte: Baja California, Baja California Sur, Chihuahua, Coahuila, Durango, Nuevo León, Sinaloa, Sonora y Tamaulipas. ${ }^{16}$ 
EDUARDO TORRE-CANTALAPIEDRA JOSÉ CARLOS YEE-QUINTERO

\section{Revisiones migratorias}

Las autoridades migratorias mexicanas realizan labores de vigilancia en el territorio nacional para identificar y poner a disposición a extranjeros que transitan por el mismo en situación irregular, las cuales son denominadas revisiones migratorias. Así, “en el periodo de 2010-2014, el INM realizó 81,294 revisiones migratorias, con las cuales identificó a 214,727 extranjeros en situación migratoria irregular" (Auditoría Superior de la Federación, 2014: 28). El número de revisiones migratorias realizadas — hayan sido programadas o no- da un acercamiento a la intensidad con la que las políticas inmigratorias de contención se están implementando en las diferentes regiones del territorio nacional. Se debe aclarar que la relación existente entre el número de revisiones y el número de puestas a disposición, y por ende de eventos de presentación en las estaciones migratorias realizados, no es constante, sino que la "eficacia" varía de una entidad federativa a otra.

Asimismo, las puestas a disposición realizadas por medio de revisiones migratorias pueden ser desde el cero hasta el cien por ciento de las puestas a disposición llevadas a cabo en una entidad federativa. En el Cuadro l se muestra cómo, mientras que los migrantes puestos a disposición a razón de revisiones migratorias en la región Norte suponen aproximadamente la mitad de las personas puestas a disposición por la autoridad migratoria —entre el 27 de marzo de 2013 y el 27 de marzo de 2014-, en el caso de las regiones Sur y Frontera Sur suponen alrededor del 90\%. Para las 32 entidades, esto es, para todo México, las revisiones migratorias constituyen por encima del $80 \%$ de las puestas a disposición realizadas, lo que es un indicador de la relevancia de este mecanismo de detección y puesta a disposición, que tiene entre sus consecuencias más comunes el alojamiento del migrante en una estación migratoria bajo un acto administrativo de presentación (ver Cuadro l).

La Gráfica l muestra pocos cambios en el volumen anual de revisiones migratorias en las cuatro regiones ${ }^{17}$ entre 2011 y 2013. Entre 2013 y 2015 se produjo un auge
MÉXICO ¿UNA FRONTERA VERTICAL? POLÍTICAS DE CONTROL DEL TRÁNSITO MIGRATORIO IRREGULAR Y SUS RESULTADOS, 2007-2016

en todas ellas, pero particularmente en las regiones Sur y Frontera Sur, reportándose el mayor aumento entre 2014 y 2015, en coincidencia con la ejecución del Plan Frontera Sur. Entre 2015 y 2016 las cifras de las regiones Norte y Centro continuaron en un paulatino ascenso, mientras que las de las regiones Sur y Frontera Sur cayeron. Por tanto, las cifras globales estarían revelando un importante auge en la tarea de contención hacia el año 2015 - con aproximadamente 40000 revisiones migratorias, duplicando las del año 2011-; este brusco auge quedó revertido en buena medida un año después con una importante caída —un descenso de alrededor de 12000 revisiones(ver Gráfica 1). ${ }^{18}$

La Gráfica 2 ofrece una mejor perspectiva de cómo se distribuye el esfuerzo de detectar inmigrantes indocumentados a través del territorio del país. Lo primero que llama la atención es que la distribución por regiones de las revisiones migratorias en 2010 difiere sustancialmente de la de los demás años. Esto puede deberse a cambios muy sustanciales en la implementación, pero también puede reflejar problemas en el proceso de registro y recopilación de la información sobre esta clase de actuaciones. Destaca especialmente el hecho de que el número de revisiones migratorias en la Frontera Sur sea tan reducido en comparación con las otras regiones (ver Gráfica 2).

La situación en los demás años apunta hacia algo distinto. De 2011 a 2016 la región en la que se realizó un mayor número de revisiones fue la Frontera Sur, donde se condujeron el $45 \%$ de las revisiones realizadas en México los dos primeros años, y algo más de una tercera parte los años siguientes. Esto quiere decir que en los últimos cuatro años cerca de dos terceras partes de las revisiones migratorias se realizaron en entidades federativas no fronterizas con Belice y Guatemala. Además, destaca el hecho de que el número de revisiones migratorias realizadas en la región Norte experimentó un crecimiento sustancial entre 2011 y 2016, de modo que se duplicó su porcentaje en el total —de un $12.4 \%$ a un $25.6 \%$ - Por tanto, es posible concluir que se ha progresado hacia una cada vez mayor igualdad en el número de revisiones 
migratorias efectuadas entre las regiones; en otras palabras, se estaría produciendo una "verticalización" de la frontera en términos de revisiones migratorias.

\section{Presentaciones ante las autoridades migratorias}

Una medida para entender la eficacia de la política migratoria en la labor de contención de los flujos migratorios son las cifras de presentaciones ante las autoridades migratorias - aseguramientos-.

Las Gráficas 3 y 4 permiten un acercamiento cuantitativo a la importancia de los primeros filtros realizados en la Frontera Sur. En tal región se producen el grueso de las presentaciones, por más que la política esté verticalizada, lo que se debe a la concentración del flujo de transmigrantes y a la captación de migrantes establecidos o con mecánicas commuters. Aunque el número de presentaciones en dicha región puede variar entre las 40000 y las 120000 personas, en ella se llevan a cabo más de la mitad de las presentaciones que ocurren en todo el país. Asimismo, en todas las regiones se puede apreciar un incremento de los eventos de presentación a partir del año 201l, el cual fue más pronunciado en las regiones Frontera Sur y Sur que en las regiones Norte y Centro. El número de presentaciones en estas últimas se mantuvo relativamente constante en toda la década, con un porcentaje en torno al 12\% de los eventos anuales en cada una de ellas (ver Gráficas 3 y 4 ).

La región Sur muestra un auge continuo en el número de presentaciones desde el año 2012, coincidiendo con el cambio de gobierno y la llegada de Peña Nieto a la presidencia (2012-2018). Dado que el número de presentaciones en las demás regiones presenta una tendencia hacia la baja, esto supone un aumento de su importancia relativa - pasó de una cuota de $14.9 \%$ en 2012, al 26.9\% en 2013-. Esta mayor "verticalidad" en lo que respecta a las presentaciones que se alcanzó en 2013 se fue revirtiendo año tras año, de tal modo que en 2016 la situación se asemejaba a la de 2012.

Se debe tener en cuenta que las dificultades para detener a los migrantes indocumentados por parte de las autoridades difieren sustancialmente de una entidad a otra, de manera que el esfuerzo de las políticas de contención en cada entidad federativa no se refleja de igual manera en las cifras de presentaciones.

\section{Estaciones migratorias y estancias provisionales}

Como se puede apreciar en la primera parte de este artículo, la estrategia mexicana de contención de los flujos se apoyó en el establecimiento de estaciones migratorias $^{19}$ y de estancias provisionales ${ }^{20}$ a lo largo del territorio. Con tales establecimientos el gobierno logró disponer del espacio necesario para alojar a los migrantes irregulares presentados para posteriormente, en la mayoría de los casos, proceder a su deportación; destaca el hecho de que durante el sexenio de Fox Quesada (2000-2006) se produjo un enorme despliegue de esta clase de instalaciones a lo largo del territorio. Este apartado se centra en el análisis de la distribución de estos establecimientos, destinados a alojar inmigrantes indocumentados, a lo largo del territorio.

En el Cuadro 2 se muestra cómo las estaciones migratorias de la región Frontera Sur suponen el 38.2\% de la capacidad total de todas las estaciones, seguida por las de la región Sur con el 25.4\%, Centro con el 20.2\% y Norte con el 16.2\%. En otras palabras, cuanto más lejos de la frontera con Belice y Guatemala, menor es la capacidad de las estaciones de una región. Por su parte, las estancias provisionales, que poseen una capacidad muy inferior que las estaciones migratorias, 660 frente a 3705 migrantes, presentan una mayor concentración en la Frontera Sur -un 60\% - (ver Cuadro 2).

La Gráfica 5 muestra la capacidad de las instalaciones de ambos tipos para alojar migrantes en cada región. El resultado es que cuatro de cada diez lugares para alojar migrantes se concentran en la región Frontera Sur, mientras que los restantes se distribuyen de manera más equitativa. En suma, puede observarse que la distribución de las estaciones migratorias y estancias provisionales apoya la idea de la existencia de una frontera vertical en el territorio mexicano (ver Gráfica 5). 
EDUARDO TORRE-CANTALAPIEDRA JOSÉ CARLOS YEE-QUINTERO

\section{Reflexiones finales}

Desde comienzos de siglo XXI, y en concreto a lo largo del sexenio de Fox Quesada, se estableció una estrategia general de contención de los flujos migratorios en la Frontera Sur y a lo largo de las rutas por las que transitan los migrantes, para lo cual se establecieron aproximadamente medio centenar de estaciones en todo el territorio nacional. El análisis de las actuaciones del gobierno, de las cifras de revisiones migratorias y de presentados parece indicar que, en líneas generales, la estrategia no ha cambiado desde entonces.

Efectivamente, el gobierno mexicano ha erigido una "frontera vertical" que pone a los migrantes en riesgo continuo de ser detectados en territorio mexicano, y posteriormente deportados. Las cifras de revisiones migratorias y de presentaciones, junto con las de capacidad de las estaciones migratorias y provinciales, también así lo atestiguan. Las cifras de revisiones migratorias y de presentaciones apuntan a que los recursos utilizados para la implementación de la política son fundamentales en los resultados. Asimismo, permiten ver un contraste entre la "verticalidad" de las revisiones migratorias, que se distribuyen de forma más equitativa por el territorio nacional, y la "menor verticalidad" de las presentaciones, que se concentran principalmente en la región Frontera Sur. Es importante considerar que esto puede derivar de procesos internos del INM; en el desconocimiento de mecanismos juega un papel importante la existencia ornamental de los métodos de transparencia.

En el último decenio, el énfasis de la política migratoria se ha puesto en el respeto a los derechos humanos de los migrantes y en la seguridad nacional. Ambos paradigmas no son incompatibles, pero en la práctica ha primado el segundo respecto al primero. Una mirada de conjunto a la bibliografía recogida en este trabajo referente a la relación entre el Estado, los abusos y violencia en contra de los migrantes y la vulneración de sus derechos permite compendiar cinco dilemas persistentes: 1) por acción o por omisión del Estado, los migrantes indocumentados en tránsito han sufrido abusos y violencia; 2) las políticas mexicanas de
MÉXICO ¿UNA FRONTERA VERTICAL? POLÍTICAS DE CONTROL DEL TRÁNSITO MIGRATORIO IRREGULAR Y SUS RESULTADOS, 2007-2016

control de los flujos migratorios han incrementado los riesgos que implica el tránsito indocumentado por el territorio nacional; 3) existe un déficit de información cuantitativa con respecto a los abusos y a la violencia que los migrantes indocumentados padecen; 4) falta de transparencia y de rendición de cuentas de las actuaciones conducidas por el Estado respecto a la migración en tránsito y limitada capacidad para sancionar los abusos cometidos, y 5) gran dificultad para realizar evaluaciones precisas sobre la implementación de las políticas de contención migratoria en México.

Una pregunta que queda pendiente para futuras investigaciones es si se producirán cambios en la política relacionados con el tránsito de migrantes por el país en la era de las políticas antiinmigración del presidente estadounidense Donald Trump. Se ha señalado que México ha seguido una estrategia migratoria y de seguridad inspirada en la de Estados Unidos, y que este último país ha influido en la política de contención de la población centroamericana en tránsito por México. Dado el actual clima de relaciones entre ambos países, algunas voces se han alzado para señalar que México debería desmarcarse de las políticas migratorias de Estados Unidos. Sin embargo, se debe tener en cuenta que existen otros factores que compelen al gobierno mexicano a llevar una política de control. Así, a México, como Estado-nación, se le exige el control de la población en su territorio, así como de los flujos migratorios que entran y salen a través de sus fronteras, actuaciones que deben realizarse no sólo de acuerdo con las necesidades del Estado, sino en aras de la protección de los derechos humanos de los migrantes.

\section{Notas}

${ }^{1}$ Agradecemos muy sinceramente a Rodolfo Casillas, a Manuel Ángel Castillo, a Gretchen Kuhner y a Ernesto Rodríguez por los consejos y sugerencias para la elaboración de este trabajo. Agradecemos a Iván Chavira por su apoyo para la realización de las solicitudes de información a través de la Plataforma de Nacional de Transparencia de México. Una versión anterior de este trabajo fue presentada en el Eighth 
Annual Transnational Latino Conference Transnational Migration: Border Crossing and Violence, celebrado del 19 al 20 de mayo de 2017 en Morelia, organizado por la DePaul University, la Universidad Michoacana de San Nicolás de Hidalgo y la Universidad Nacional Autónoma de México, campus Morelia.

${ }^{2}$ Ver, por ejemplo, Sánchez (1993), CNDH (1996, 2009), Casillas (2002, 2007, 2012, 2016), García y Tarrío (2008), Insyde (2013), WOLA (2014), Silva Quiroz $(2014,2015)$ y Yee $(2016)$.

${ }^{3}$ Utilizando la compleja, y en muchas ocasiones confusa, terminología jurídico-administrativa actual se diría que: los migrantes se exponen a ser "detectados y puestos a disposición" de las autoridades migratorias, "presentados" -ingresados en una estación migratoria mediante el procedimiento administrativo de presentación, por no haber acreditado su situación migratoria según lo previsto por Ley de Migración 2011 y su Reglamento 2012; anteriormente, asegurados-, "alojados" en una estación migratoria y "deportados" o, en su caso, expulsados mediante el llamado "retorno asistido".

${ }^{4}$ Ver, por ejemplo: Anguiano (2008), Jácome (2008), González Velázquez (2011), Insyde (2013), García y Villafuerte (2014), WOLA (2014), Martínez, Cobo y Narváez (2015), Silva Hernández (2015), Villafuerte y García (2015), Díaz (2016), Torre y Schiavon (2016), Yee y Torre (2016).

${ }^{5}$ Se seleccionó este grupo poblacional porque incluye la inmensa mayoría de los migrantes en tránsito irregular por México; suponen más del $80 \%$ de los presentados en estaciones migratorias en 2016 —estimaciones propias con base en los boletines del INM, 20082017- Sin embargo, no se desconoce el creciente número de migrantes indocumentados en tránsito procedentes de diversos países latinoamericanos y de otros continentales como los flujos de haitianos que llegaron a Tijuana y a otras ciudades fronterizas desde mediados de 2016

${ }^{6}$ Se analizaron los primeros cuatro años de este sexenio.

${ }^{7}$ Disponible en: http://www.plataformadetransparencia. org.mx/web/guest/inicio

${ }^{8}$ Las denominadas "presentaciones ante la autoridad migratoria" - "asegurados" con anterioridad a la Ley de Migración de 2011 - son eventos de entrega de extranjeros a centros de detención del INM por no acreditar administrativamente su estancia legal en México. Tal como lo dicta el artículo 99 de la Ley de Migración: "Es de orden público la presentación de los extranjeros en estaciones migratorias o en lugares habilitados para ello, en tanto se determina su situación migratoria en territorio nacional. La presentación de extranjeros es la medida dictada por el Instituto mediante la cual se acuerda el alojamiento temporal de un extranjero que no acredita su situación migratoria para la regularización de su estancia o la asistencia para el retorno". La mayoría son posteriormente deportados.

${ }^{9}$ Se hace notar que los registros administrativos en el siglo pasado fueron muy deficitarios, por lo que, además del hecho de que son eventos y no personas los que quedan registrados debe entenderse que los datos de los flujos migratorios son aproximados.

${ }^{10}$ Define transmigrante como aquel: "en tránsito hacia otro país y que podrá permanecer en territorio nacional hasta por treinta días" (Congreso de la Unión, 1974: cap. 3, art. 41).

${ }^{11}$ Casillas (2016) indica que esto se debe: 1) al pequeño tamaño del flujo y su discontinuidad, 2) a que se pensó que la migración se detendría cuando finalizaran los conflictos políticos en los países de origen, y 3) a que era un flujo diferenciable del migratorio laboral en la región fronteriza.

${ }^{12}$ Para un análisis en detalle del Plan Sur, ver Casillas (2002).

${ }^{13}$ Ver, por ejemplo, CNDH (1996).

${ }^{14}$ Ribando Seelke, Clare y Kristin Finklea (2017). U.S. Mexican Security Cooperation: The Mérida Initiative and Beyond. Disponible en: https://fas.org/sgp/crs/row/ R41349.pdf

${ }^{15}$ Ver, por ejemplo: CNDH (2009), Insyde (2013), CIDH y OEA (2013) y WOLA (2014).

${ }^{16}$ Esta clasificación se construyó con base en tres criterios: 1) la distancia de la entidad federativa respecto a la Frontera Sur, 2) si se trata de entidades federativas fronterizas tanto con Belice y Guatemala, como con Estados Unidos, y 3) las rutas migratorias. 
17 Como respuesta a dos consultas realizadas en la Plataforma Nacional de Transparencia de México se obtuvieron datos de las revisiones migratorias de los años 2010 a 2016.

${ }^{18}$ Las cifras de revisiones migratorias totales realizadas en México son: 18016 en 2010; 20220 en 2011; 20786 en 2012; 20856 en 2013; 25383 en 2014, 40964 en 2015; y 28481 en 2016; datos obtenidos de la Plataforma Nacional de Transparencia.

${ }^{19}$ Se denomina estación migratoria: "a la instalación física que establece el Instituto para alojar temporalmente a los extranjeros que no acrediten su situación migratoria regular, en tanto se resuelve su situación migratoria" (Congreso de la Unión, 2017: art. 3, X).

20 "Las estancias provisionales son aquellas instalaciones físicas que el Instituto establece o habilita para alojar de manera provisional a las personas extranjeras que no acrediten su situación migratoria regular, hasta en tanto sean trasladados a una Estación Migratoria o sea resuelta su situación migratoria en términos de la Ley y su Reglamento. Dichas Estancias de acuerdo con sus características físicas se clasifican en: I. Estancias provisionales $\mathrm{A}$, que permiten una estancia máxima de cuarenta y ocho horas, y II. Estancias provisionales B, que permiten una estancia máxima de siete días" (Secretaría de Gobernación, 2012: art. 5).

\section{Referencias}

Alba, Francisco y Manuel Ángel Castillo (2012). New Approaches to Migration Management in Mexico and Central America. Washington D.C.: Migration Policy Institute. Disponible en: https://bit.ly/2L8vDnP (consultado el 15 de agosto de 2017).

Anguiano Téllez, María Eugenia (2008). “Inmigración, emigración y tránsito migratorio en Chiapas: Un bosquejo general". En LiminaR. Estudios Sociales y Humanísticos, VI(2): 142-154.

Anguiano Téllez, María Eugenia y Alma Trejo Peña (2007). "Políticas de seguridad fronteriza y nuevas rutas de movilidad de migrantes mexicanos y guatemaltecos". En LiminaR. Estudios Sociales y Humanísticos, V(5): 47-65.
Auditoría Superior de la Federación (2014). Informe del resultado de la fiscalización superior de la cuenta pública 2014: auditoría de desempeño: 14-0-04K00-07-0060 GB-022. Disponible en: https://bit.ly/2IubCGH (consultado el 15 de agosto de 2017).

Baggio, Fabio (2010). "Fronteras nacionales, internalizadas y externalizadas". En María Eugenia Anguiano Téllez y Ana María López Sala (eds.). Migraciones y fronteras. Barcelona: Icaria-CIDOB, pp. 49-73.

Casillas R., Rodolfo (2002). "El Plan Sur de México y sus efectos sobre la migración internacional”. Ecuador Debate, 56: 199-210.

Casillas R., Rodolfo (2007). Una vida discreta, fugaz y anónima: Los centroamericanos transmigrantes en México. México: CNDH y OIM.

Casillas R., Rodolfo (2012). “Construcción del dato oficial y realidad institucional del flujo indocumentado en los registros del INM". En Migración y Desarrollo, 10(19): 33-60.

Casillas R., Rodolfo (2016). Entre la política deseada, la practicada y los flujos migratorios emergentes: respuestas en construcción y desafíos duraderos. Documento de trabajo 4. Migración en tránsito-ITAM.

Castillo, Manuel Ángel (2001). "Tendencias y Determinantes Estructurales de la Migración Internacional en Centroamérica." En Luis Rosero Bixby (ed.), Población del Istmo 2000: familia, migración, violencia y medio ambiente. San José, Costa Rica: Centro Centroamericano de Población de la Universidad de Costa Rica, pp. 187-209.

Castillo, Manuel Ángel (2005). "Fronteras, migración y seguridad en México”. En Alteridades, 15(30): 51-60.

CIDH y OEA (2013). Derechos humanos de los migrantes $y$ otras personas en el contexto de la movilidad humana en México. S.l.: CIDH, OEA. Disponible en: https://bit. ly/lpFp90I (consultado el 15 de agosto de 2017).

CNDH (1996). Informe sobre violaciones a los derechos humanos de los inmigrantes. Frontera Sur. México: $\mathrm{CNDH}$.

CNDH (2009). Informe especial sobre los casos de secuestro en contra de migrantes. México: CNDH. Disponible en: https://bit.ly/2oh9FTu (consultado el 20 de diciembre de 2016). 
Congreso de la Unión de los Estados Unidos Mexicanos (1974). Ley General dePoblación, 1974, y reformasposteriores. Disponible en: https://bit.ly/UsDUqX (consultado el 16 de agosto de 2017).

Congreso de la Unión de los Estados Unidos Mexicanos (2017). Ley de Migración, 2011. Disponible en: http:// www.diputados.gob.mx/LeyesBiblio/ref/lmigra.htm (consultado el 16 de agosto de 2017).

Díaz Prieto, Gabriela (2016). Operativos móviles de revisión migratoria en las carreteras de México. Una práctica discriminatoriaeilegal. Tijuana: El Colegio de la Frontera Norte. Disponible en: https://bit.ly/2IUd0FM (consultado el 15 de mayo de 2017).

Espino Tapia, Diana Rocío y Juan Antonio Doncel de la Colina (2016). "Impacto de la legislación en materia de migración en la realidad social de inmigrantes centroamericanos en situación irregular en la ciudad de Monterrey (México)". En Revista de Derecho Público, (36): 1-24. Disponible en: https://bit.ly/2wOuVZP (consultado el 16 de mayo de 2017).

García Aguilar, María del Carmen y Daniel Villafuerte Solís (2014). Migración, derechos humanos y desarrollo. Aproximaciones desde el sur de México y Centroamérica. México: Universidad de Ciencias y Artes de Chiapas/ Juan Pablos Editor.

García Aguilar, María del Carmen y María Tarrío García (2008). "Migración irregular centroamericana. Las tensiones entre derechos humanos, ley y justicia". En Daniel Villafuerte Solís y María del Carmen García Aguilar (coords.), Migraciones en el sur de México y Centroamérica. México: Universidad de Ciencias y Artes de Chiapas, Miguel Ángel Porrúa, pp. 123-170.

Gobierno de los Estados Unidos Mexicanos, Presidencia de la República (2007). Plan Nacional de Desarrollo 2007 2012. Disponible en: http://pnd.calderon.presidencia. gob.mx/ (consultado el 15 de mayo de 2017).

Gobierno de los Estados Unidos Mexicanos, Presidencia de la República (2013). Plan Nacional de Desarrollo 2013-2018. Disponible en: https://bit.ly/Q4xGLA (consultado el 15 de mayo de 2017).

González Velázquez, Eduardo (2011). Frontera vertical: México frente a los migrantes centroamericanos. México: Centro Universitario UTEG.
González-Murphy, Laura V. y Rey Koslowski (2011). Entendiendo el cambio a las leyes de inmigración de México. Woodrow Wilson International Center for Scholars Mexican Institute. Disponible en: https://bit. ly/2rSN2YG (consultado el 4 de febrero de 2018).

Instituto Nacional de Migración. Centro de Estudios Migratorios (2005). Propuesta de políticas migratoria integral en la frontera sur de México. México: Secretaría de Gobernación / INM. Disponible en: https://bit. ly/2wPaifY (consultado el 15 de mayo de 2017).

Instituto Nacional de Migración (2008-2017). Boletines mensuales de Estadísticas Migratorias, 2007-2016. México: SEGOB e INM. Disponibles en: http://www. politicamigratoria.gob.mx/es_mx/SEGOB/Boletines_ Estadisticos (consultados el 10 de julio de 2017).

Insyde (2013). Diagnóstico del Instituto Nacional de Migración: hacia un sistema de rendición de cuentas en pro de los derechos de las personas migrantes en México. Disponible en: http:// insyde.org.mx/diagnosticodelinm (consultado el 15 de agosto de 2017).

Jácome, Felipe (2008). "Trans-Mexican Migration: A Case of Structural Violence", working paper, Georgetown University / Center for Latin American Studies. Disponible en: http://pdba.georgetown.edu/ CLAS\%20RESEARCH/Working\%20Papers/WP2. pdf (consultado el 25 de enero de 2018).

Martínez, Fabiola y Gustavo Castillo (2014) "Anuncian puntos de control en la frontera sur para acciones precisas contra criminales". En La Jornada, 25 de marzo. Disponible en: http://www.jornada.unam. mx/2014/03/25/politica/013nlpol (consultado el 19 de agosto de 2017).

Martínez, Graciela, Salvador David Cobo y Juan Carlos Narváez (2015). "Trazando rutas de la migración de tránsito irregular o no documentada por México". En Perfiles Latinoamericanos, 23(45): 127-155.

Sánchez Munguía, Vicente (1993). "Matamoros-sur de Texas: el tránsito de los migrantes de América Central por la frontera México-Estados Unidos". En Estudios Sociológicos, XI(31): 183-207.

Secretaría de Gobernación (2012). Acuerdo por el que se emiten las Normas para el funcionamiento de las Estaciones Migratorias y Estancias Provisionales del Instituto Nacional 
de Migración. Disponible en: http://dof.gob.mx/ nota_detalle.php?codigo=5276965\&fecha $=08 / 11 / 2012$ (consultado el 19 de agosto de 2017).

Silva Hernández, Aída (2015). "Estrategias de tránsito de adolescentes centroamericanos independientes: enfrentando la frontera vertical en México". En REMHU: Revista Interdisciplinar da Mobilidade Humana, (44): 99-117.

Silva Quiroz, Yolanda (2014). Transmigración de centroamericanos por México: su vulnerabilidad y sus derechos humanos. Tesis de doctorado. El Colegio de la Frontera Norte.

Silva Quiroz, Yolanda (2015). "Reflexiones en torno al control migratorio y los derechos humanos en México". En Revista Legislativa de Estudios Sociales y de Opinión Pública, 8(15): 43-74.

Torre Cantalapiedra, Eduardo y Jorge A. Schiavon (2016). "Actuar o no actuar: un análisis comparativo del rol de los estados de Chiapas y Arizona en la gestión de la inmigración”. En Norteamérica, 11(1): 159-189.

Urbano Reyes, Francisco (2012). "La gestión de Felipe Calderón en materia de migración: sin comienzo y sin final". En Sexenio en perspectiva. Análisis académico del gobierno de Felipe Calderón, Ciudad de México: Universidad Iberoamericana, pp. 77-87.
Velázquez Flores, Rafael y Roberto Domínguez (2013). "Balance de la política exterior de México en el sexenio de Felipe Calderón bajo los tres niveles de análisis: límites y alcances". En Foro Internacional, 53(213-214): 483-516.

Villafuerte Solís, Daniel y María del Carmen García Aguilar (2015). "Crisis del sistema migratorio y seguridad en las fronteras norte y sur de México". En REMHU: Revista Interdisciplinar da Mobilidade Humana, 23(44): 83-98.

WOLA (2014). Laotra fronterade México. Seguridad, migración y la crisis humanitaria en la línea con Centroamérica. Disponible en: https:/www.wola.org/sites/default/ files/La\%20otra\%20frontera.pdf (consultado el 15 de agosto de 2017).

Yee Quintero, José Carlos (2016). Las víctimas del Estado: Violencia hacia migrantes hondureños, perpetrada por agentes del Estado, durante su tránsito por México. Tesis de Maestría. México: El Colegio de la Frontera Norte.

Yee Quintero, José Carlos y Eduardo Torre Cantalapiedra (2016). "Lidiando con la frontera vertical: estrategias migratorias de los hondureños en tránsito por México". En REMHU: Revista Interdisciplinar da Mobilidade Humana, XXIV(47): 97-114. 
Cuadro 1. Volumen absoluto y relativo de migrantes puestos a disposición, entre 27 de marzo de 2013 a 27 de marzo de 2014

\begin{tabular}{cccc}
\hline Regiones & $\begin{array}{c}\text { Total migrantes } \\
\text { puestos a disposición }\end{array}$ & $\begin{array}{c}\text { Migrantes identificados puestos a } \\
\text { disposición por revisiones migratorias }\end{array}$ & $\%$ \\
\hline Norte & 8107 & 4280 & 52.8 \\
Centro & 8711 & 6079 & 69.8 \\
Sur & 25499 & 22706 & 89.0 \\
Frontera Sur & 33874 & 30012 & 88.6 \\
\hline Total & 76191 & 63077 & 82.8 \\
\hline
\end{tabular}

Fuente: elaboración propia con base en datos obtenidos a través de la Plataforma Nacional de Transparencia de México. Solicitud de información: 411100024114.

Cuadro 2. Número y capacidad de estaciones migratorias y estancias provisionales en México por regiones, octubre 2016

\begin{tabular}{crrrrrc}
\hline \multirow{2}{*}{ Regiones } & \multicolumn{3}{c}{ EM } & \multicolumn{3}{c}{ EP } \\
\cline { 2 - 7 } & $\mathbf{N}^{\circ}$ & Cap. & \% Cap. & $\mathbf{N}^{\circ}$ & Cap. & $\%$ Cap. \\
\hline Norte & 12 & 601 & 16.2 & 7 & 97 & 14.7 \\
Centro & 9 & 748 & 20.2 & 4 & 43 & 6.5 \\
Sur & 4 & 941 & 25.4 & 4 & 124 & 18.8 \\
Frontera Sur & 7 & 1415 & 38.2 & 11 & 396 & 60.0 \\
\hline Total & 32 & 3705 & 100.0 & 26 & 660 & 100.0 \\
\hline
\end{tabular}

Fuente: elaboración propia con base en datos obtenidos a través de la Plataforma Nacional de Transparencia de México. Solicitudes de información: 0411100081816 y 411100081916.

Nota: el rubro EP incluye tanto las estancias provisionales tipo A como tipo B. 
Gráfica 1. Distribución por regiones de las revisiones migratorias realizadas por la autoridad mexicana, 2010-2016

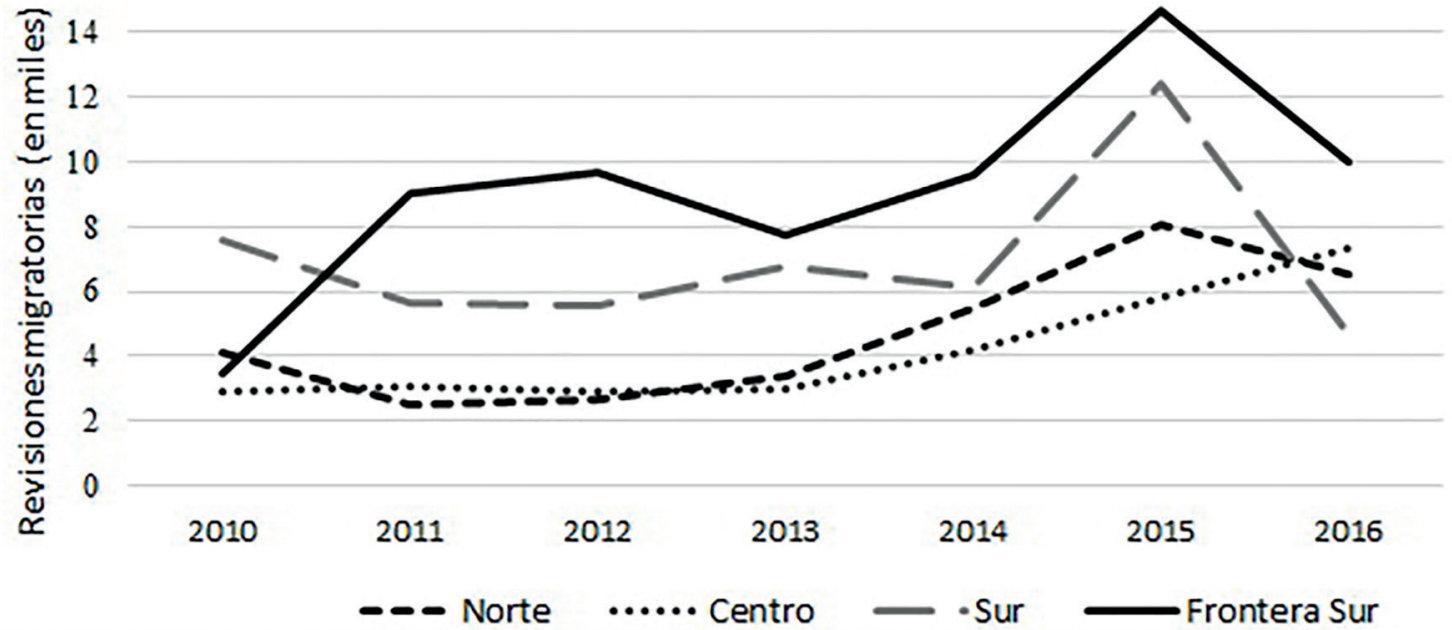

Fuente: elaboración propia con base en datos obtenidos a través de la Plataforma Nacional de Transparencia. Solicitudes de información: 0411100085013 y 0411100003417.

Gráfica 2. Distribución porcentual por regiones de las revisiones migratorias realizadas por la autoridad mexicana, 2010-2016

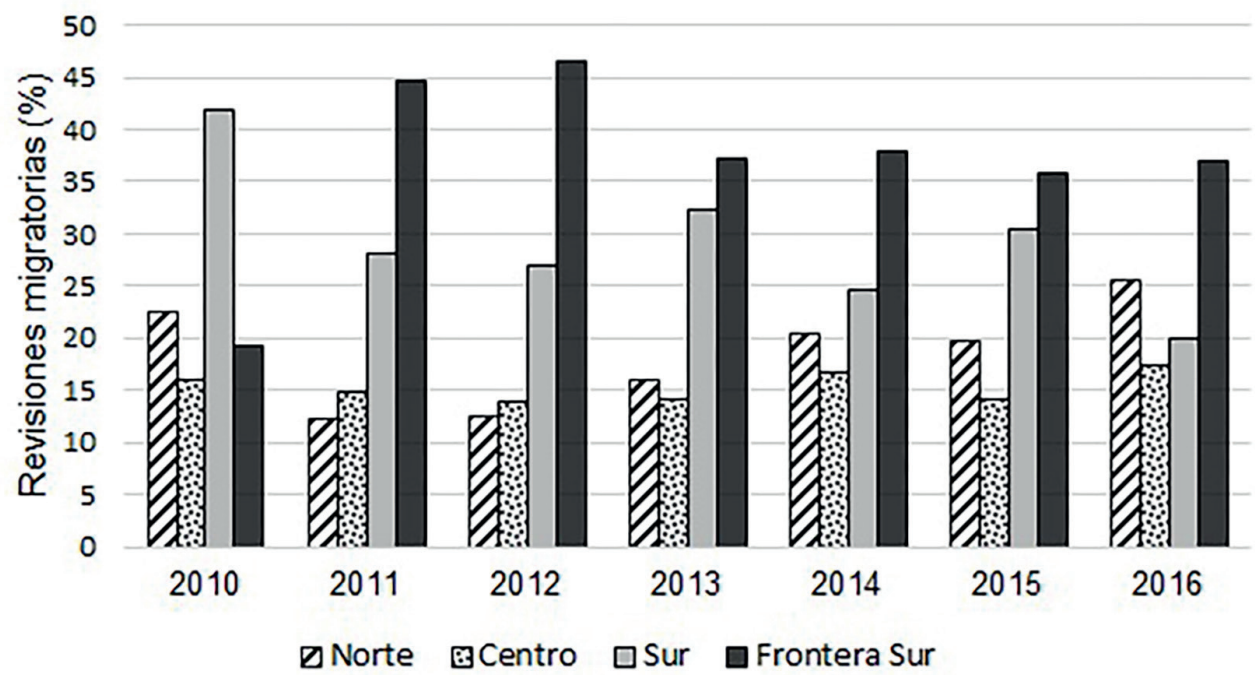

Fuente: elaboración propia con base en datos obtenidos a través de la Plataforma Nacional de Transparencia de México. Solicitudes de información: 0411100085013 y 0411100003417. 


\section{Gráfica 3. Distribución por regiones de eventos de presentación de migrantes}

ante la autoridad mexicana, 2007-2016

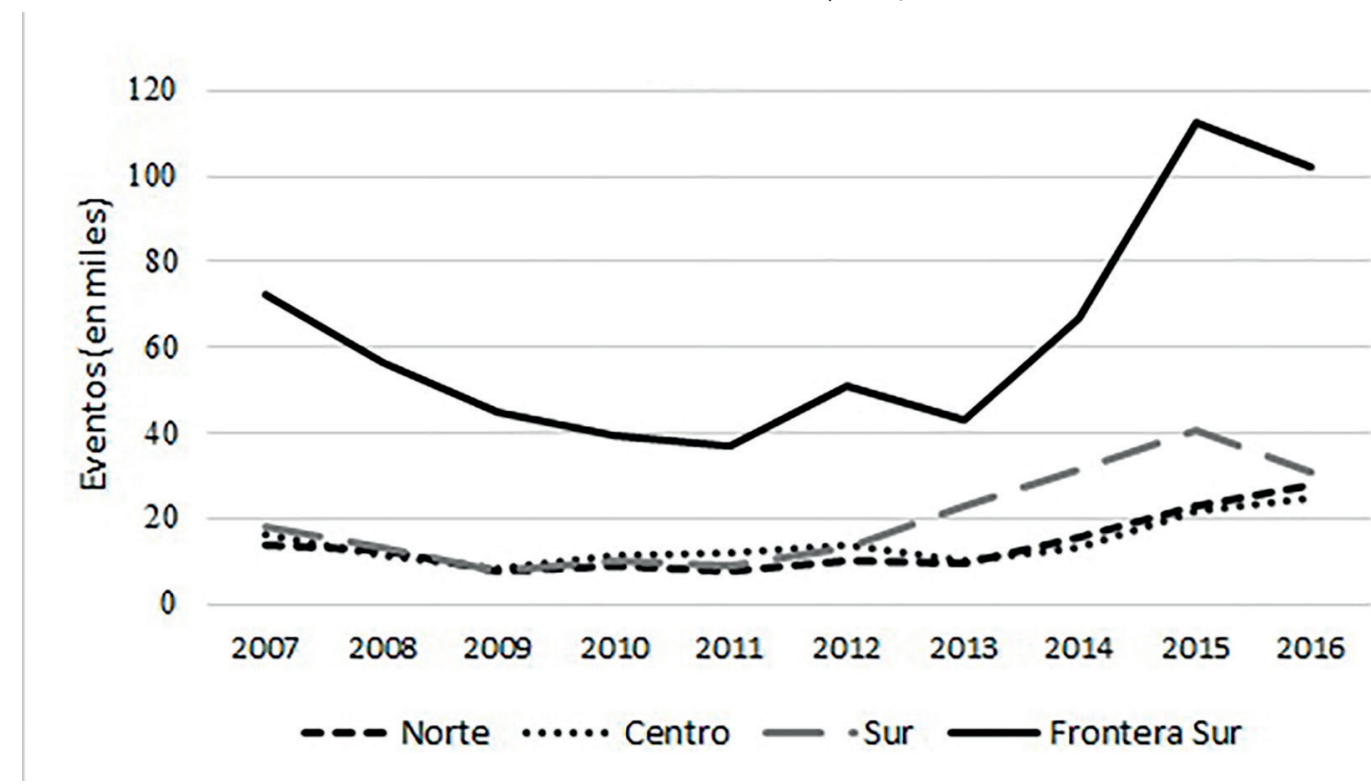

Fuente: elaboración propia con base en las estadísticas INM, 2008-2017.

Nota: a pesar de que las cifras responden primordialmente a eventos de privación de libertad de migrantes mediante un acto de presentación —anteriormente "aseguramiento"-, que implica su presencia física en estaciones y estancias migratorias, deben tenerse algunas consideraciones sobre la construcción de éstas año tras año.

- 2007 a 2011: La cifra incluye "Eventos de alojados por aseguramiento" y "Eventos de Centroamericanos acogidos al Memorándum de repatriación" (alojados para repatriación voluntaria).

2012: La cifra incluye "Extranjeros ingresados en las estaciones migratorias del INM bajo el procedimiento administrativo de presentación" y "Centroamericanos acogidos al Memorándum".

- 2013 a 2016: La cifra incluye "Eventos de migrantes ingresados en las estaciones migratorias del INM bajo el procedimiento administrativo de 'presentación' por no acreditar su situación migratoria”. Por facilidad en la lectura nos referiremos a estas cifras como presentaciones. 
Gráfica 4. Distribución porcentual por regiones de eventos de presentación de migrantes ante la autoridad mexicana, 2007-2016

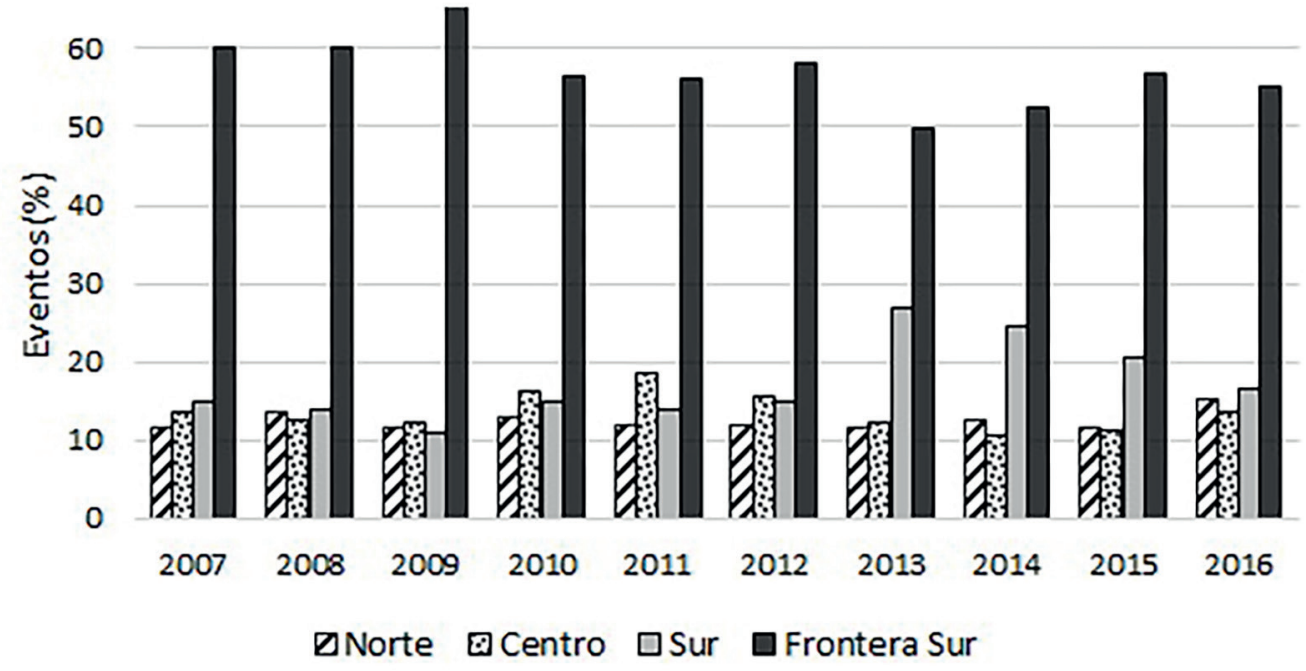

Fuente: elaboración propia con base en las estadísticas INM, 2008-2017.

Nota: a pesar de que las cifras responden primordialmente a eventos de privación de libertad de migrantes mediante un acto de presentación —anteriormente "aseguramiento"—, que implica su presencia física en estaciones y estancias migratorias, deben tenerse algunas consideraciones sobre la construcción de éstas año tras año.

Gráfica 5. Distribución porcentual de la capacidad de estaciones migratorias más las estaciones provinciales en México por regiones, octubre 2016

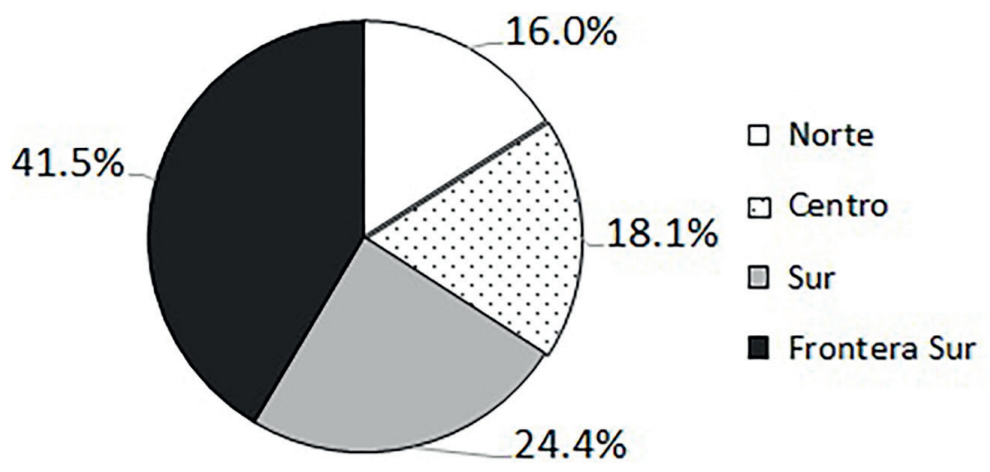

Fuente: elaboración propia con base en datos obtenidos a través de la Plataforma Nacional de Transparencia de México. Solicitudes de información: 0411100081816 y 411100081916.

Nota: el rubro EP incluye tanto las estancias provisionales tipo A como tipo B. 